\title{
Duodenogastric Reflux after Biliary Procedure
}

\author{
Naoki Hashimoto \\ Department of Surgery, Kinki University, Oosaka Sayama, Japan \\ Email: gojigen000@gmail.com
}

Received 30 July 2014; revised 12 September 2014; accepted 14 October 2014

Copyright (C) 2014 by author and OALib.

This work is licensed under the Creative Commons Attribution International License (CC BY). http://creativecommons.org/licenses/by/4.0/

(c) (i) Open Access

\section{Abstract}

Duodenogastric reflux (DGR) is a poorly understood gastrointestinal process that is defined as reflux of duodenal contents into the stomach. Therapeutic biliary procedures disrupt the function of the sphincter of Oddi. Patients are potential "bile refluxers". Methods: The present study was carried out to document the incidence and evaluate the clinical significance of DGR after cholecystectomy $(n=9)$ and choledochoduodenostomy (CDD) $(n=6)$. Duodenogastric reflux was quantified using continuous intravenous infusion of $99 \mathrm{~m}$ Tc-HIDA. They are studied by symptom evaluation and hepatobiliary scintigraphy. The scintigraphic findings were then compared with those of nine patients who had undergone cholecystectomy alone. Results: The incidence of DGR after CDD was $67 \%$ compared to $22 \%$ in the cholecystectomy alone group $(P<0.05)$. None of the patients complained of epigastric distress. Conclusion: ${ }^{99 \mathrm{~m} T c-H I D A}$ scanning of the hepatobiliary system is a reasonable and reliable method for the quantitative evaluation of DGR. CDD is frequently associated with mild to moderate DGR compared to cholecystectomy alone group. We need to understand these issues to adequately advise patients of the implications of cholecystectomy and CDD.

\section{Keywords}

Duodenogastric Reflux (DGR), Cholecystectomy, Choledochoduodenostomy (CDD), 99mTc-HIDA Hepatobiliary Scintigraphy

\section{Subject Areas: Gastroenterology \& Hepatology, Surgery \& Surgical Specialties}

\section{Introduction}

Reflux of duodenal content into the stomach, duodenogastric reflux (DGR), occurs physiologically but anything that alters the structure or function of the duodeno-pancreatobiliary system will promote DGR. Thus, surgical destruction of the pylorus after distal gastrectomy [1], pyloroplasty or whipple's procedure [2] increases DGR. DGR may occur as a natural phenomenon in healthy individuals during the early morning hours or in the post- 
prandial period. Similarly, anything that disturbs the orderly sequence of bile secretion, storage, and release may contribute to DGR [3]. The most dramatic alteration in this environment occurs after cholecystectomy and choledochoduodenostomy (CDD). Nevertheless, few studies have been conducted evaluating the incidence and severity of DGR and its clinical significance after cholecystectomy and CDD.

The conventional and the most widely accepted method of diagnosing DGR is the measurement of intragastric bile acid in the gastric juice aspirated through nasogastric tube and hepatobiliary scintigraphy. In the last few years, scintigraphic radiological techniques, such as imaging with hepatobiliary scintigraphy, have become available to study dynamic duodenogastric reflux [4].

The aim of this work is to evaluate the symptom and incidence of DGR after cholecystectomy and CDD by scintigraphy.

\section{Material and Methods}

A total of six patients who had undergone cholecystectomy and end-to-side CDD for choledocholithiasis $(\mathrm{n}=3)$ or Lemmel syndrome $(n=3)$ in this study. Nine patients who had undergone cholecystectomy alone for symptomatic cholelithiasis acting as a control to CDD group. Postoperative biliary scintigraphy was done between 6 months and a year after cholecystectomy and CDD. Prior to the scan, all drugs liable to affect the upper gut motility were stopped for 48 hours. The patients refrained from smoking and fasted for 4 - 6 hours before the scan. All patients were assessed by personal interview and scintigraphy. Their scintigraphic findings were compared with those of 9 patients who had undergone cholecystectomy alone for symptomatic cholelithiasis acting as a control to the CDD group.

\subsection{Scintigraphy}

Hepatobiliary scintigraphy was performed using $3-4 \mathrm{~m}$ Ci of ${ }^{99 \mathrm{~m}}$ Tc-HIDA. The technique of the scintigraphic evaluation has been described in detail previously [5].

The anterior abdominal images were taken with a gamma camera, each image of $500 \mathrm{~K}$ counts being taken at 5 -min intervals for $60 \mathrm{~min}$. The image interpretation and severity of DGR was assessed and graded according to the following scale: 0 , no reflux; 1 , reflux only into the antrum; 2 , moderate reflux into the body; 3 , marked reflux into the body and funds; and 4, reflux into the esophagus.

\subsection{Symptom Scoring}

The patients were evaluated by personal interview to assess the symptoms and their severity. The presence and severity of epigastric pain, nausea, and vomiting were scored according to the following scale [6]: 0 , symptoms not present; 1 , occasional symptoms; 2, symptoms requiring medical treatment; and 3, symptoms interfering with daily activity. An overall symptom score was obtained for each patient by adding the score of each symptom, with the maximum symptom score being 9.

\section{Results}

Table 1 is the summary of the results of examinations performed on patients who underwent CDD and cholecystectomy. Of the 6 patients, 4 (67\%) had varying degrees of DGR after CDD according to hepatobiliary scintigraphy. While these four patients had mild to moderate reflux, as grade 1 in 2, grade 2 in 1 patient and grade 3 in 1 patient. Figure 1(a) is a typical grade 3 of scintigraphy assessed DGR after CDD. Figure 1(b) is a typical grade 1 of scintigraphy assessed DGR after cholecystectomy. In the cholecystectomy alone group, mild DGR of grade 1 was observed in 2 (22\%) of the 9 patients. A significant difference was found between the two groups. None of the patients complained of epigastric distress.

\section{Discussion}

DGR is a natural physiological phenomenon often occurring during the early hours of the morning and postprandial period. It is commonly understood to mean the passing into the stomach of duodenal fluid containing secretions from the intestinal mucosa, bile and pancreatic fluid.

The various techniques employed to detect DGR are endoscopy gastroduodenal intubation and direct sampling, gastric $\mathrm{pH}$ monitoring, Bilitec method and hepatobiliary scintigraphy. Among them, the use of the intuba- 
Table 1. Summary of the results of examinations performed on 6 patients with CDD and 9 patients with cholecystectomy.

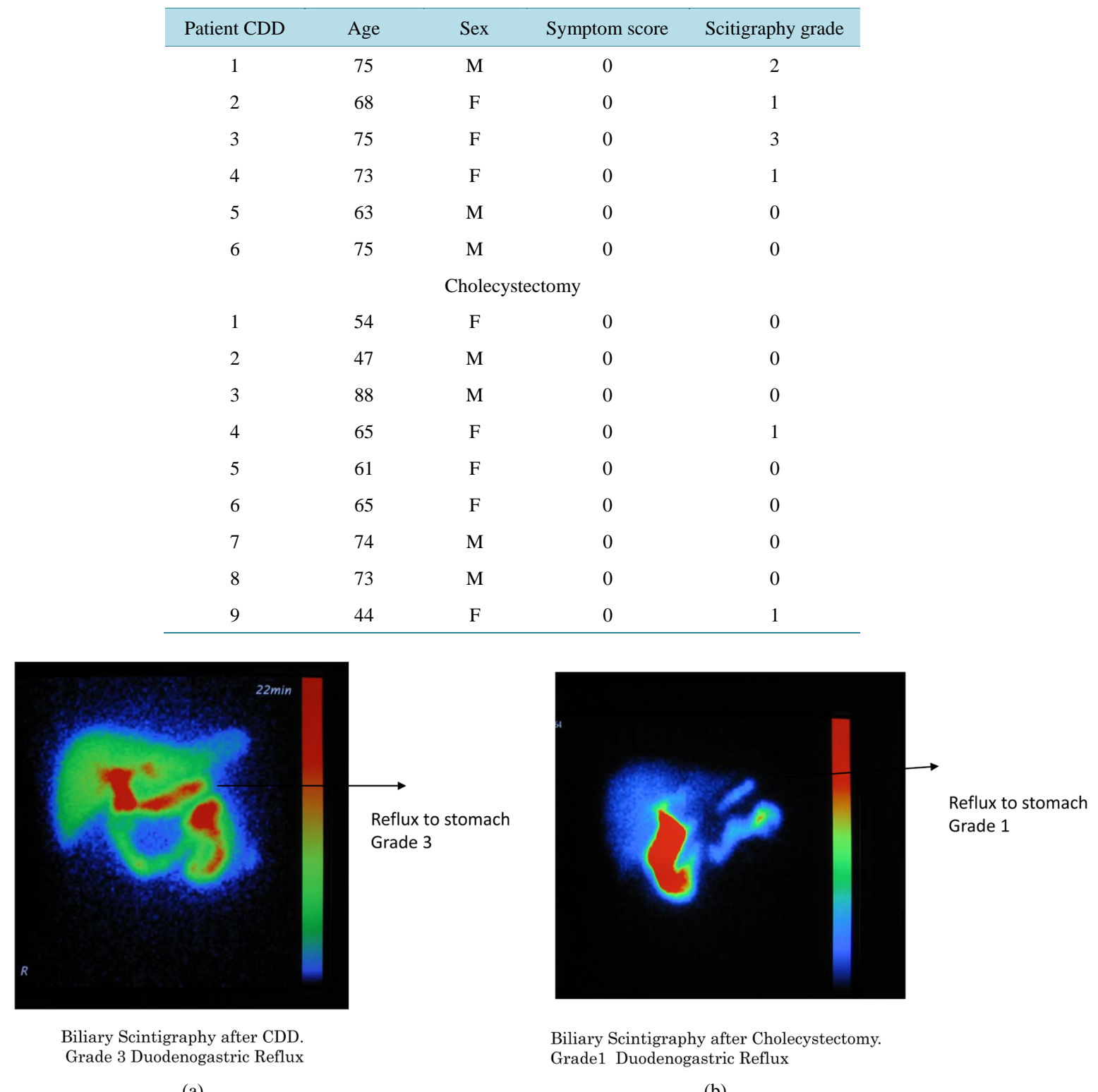

(a)

(b)

Figure 1. (a) Biliary scintigraphy after CDD. Grade 3 duodenogastric reflux; (b) Biliary scintigraphy after cholecystectomy. Grade 1 duodenogastric reflux.

tion technique is considered nonphysiologic since it is invasive and thereby may provoke reflux. Gastric pH monitoring is cumbersome, entails the use of sophisticated instruments and is uncomfortable for the patients. Scintigraphic documentation of DGR is technically easy, simple and physiologic as it is noninvasive. Bilitec method allows ambulatory detection of bile in the esophagus in much the same manner as pH detction. Nevertheless there are some limitations. Some coloured foods may interfere with the measurements and small pieces of food may get stuck between the mirror and the tip of the fiber optic bundle. At low $\mathrm{pH}$ below 3.5 bilirubin forms dimers with different optic properties causing an underestimation of bilirubin concentration of at least 30 percent [7].

Just et al. [8] showed that there was no correlation between an alkaline $\mathrm{pH}$ and the presence of bilirubin. Therefore, we used hepatobiliary scintigraphy to evaluate DGR after cholecystectomy and CDD. 
Cholecystectomy is the most frequent elective abdominal surgical operation and most patients are perfectly well afterwards. Occasionally patients have symptoms of nausea and pain but these usually settle over time. In some, biliary dyskinesia is a disturbing consequence. Thankfully, for the majority with complicated biliary pathology, this is an uncommon outcome. It is particularly important to understand the physiopathology that may arise from cholecystectomy and CDD in asymptomatic patients.

The mechanism of gallbladder function is very complex and requires many humoral and neural controls. During the interdigestive period the gallbladder is relaxed, the sphincter of Oddi is closed and the gallbladder fills with bile. Bile is stored in the gallbladder between meals and is expelled into the duodenum by contraction of gallbladder in response to meal stimulated CCK secretion from the duodenum. CCK is released in response to small peptides and fatty acids in the duodenum. It causes contraction of the gallbladder and relaxation of the sphincter of Oddi. In addition CCK causes relaxation at the lower esophageal sphincter (LOS) [9]. CCK release is switched off by the negative feedback effect of the bile bolus in the duodenum. Thus, when the gallbladder is functioning normally, bile is mixed with food in the duodenum and little is free to enter the stomach or available for contact with the esophageal mucosa [10].

The orderly sequence of bile storage and release is dramatically disturbed by cholecystectomy. When the gallbladder is removed, the facility for storage between meals is destroyed and bile flows continuously into the duodenum promoting retrograde reflux into the stomach leading to duodeno-gastric reflux. The loss of bile reservoir functions leads to an abnormal situation whereby bile flows into the duodenum in a continuous rather than intermittent fashion, and is related to food intake. As the bile is delivered constantly into the duodenum, the mechanism for switching off CCK secretion is impaired. Furthermore, as no bile bolus enters the duodenum there is no mechanism for switching off CCK secretion. CCK levels remain elevated after meals [9] and CCK may further contribute by reducing the lower esophageal pressure.

Cholecystectomy also directly alters the motor function of the gastric antrum, pylorus and duodenum. In a dog model, cholecystectomy increased the bile salt content of the stomach and the frequency of pyloric relaxation during phase of the migrating motor complex [11]. These findings have been confirmed in humans with increased fasting gastric bile acid concentrations and lowered postcibal antral motility [12].

Moreover, the main regulator of bile flow into the duodenum is the sphincter of Oddi. We consider that the surgical elimination of the function of the sphincter of Oddi, as occurs after CDD, cause a continual bile flow into the duodenum. This results in the passage of greater amounts of bile into the duodenum in the interdigestive period than after simple cholecystectomy and from there into the stomach during physiologic duodenogastric reflux. In the present study, the incidence of DGR after CDD was found to be significantly greater than that after cholecystectomy alone.

There is mounting evidence of the importance of duodenogastric reflux and this appears to be increased by CDD. There is evidence that implicates duodenogastric esophageal reflux in the pathogenesis of Barrett's esophagus and cancer [13] [14]. Walsh et al. [9] have previously shown that cholecystectomy is associated with an increased incidence of GERD. Others have shown a link between cholecystectomy and adenocarcinoma [15]. We hypothesized that cholecystectomy and CDD may contribute to duodenogastric reflux and to the metaplasiadysplasia-neoplasia sequence of esophageal cancer. These combined disturbances may contribute to an abnormal concentration of bile refluxate for a longer duration in the lower esophagus. These findings support a possible contribution of cholecystectomy and CDD in the development of esophageal cancer [16]. Patients with cholecystectomy and CDD have increased bile reflux into the esophagus and increased potential toxicity.

Duodenogastric reflux is an interesting phenomenon which has potential serious consequences. The combination of bile and acid is considered to increase the severity of esophageal damage from acid reflux and to lead to a greater incidence of Barrett's esophagus and adenocarcinoma. It is interesting that this sequence can be reproduced in a rat model [17]. Bile salts are very ionic and damage the surfactant properties of the mucus lining of the esophagus making it more sensitive to avoid damage.

Freedman et al. [16] suggested an association between cholecystectomy and an increased risk of adenocarcinoma of the esophagus, possibly due to the toxic effect of refluxed bile on esophageal mucosa. Further studies are needed regarding the link between bile reflux and esophageal carcinogenesis.

Therefore, there is mounting evidence of the importance of duodenogastric reflux and this appears to be increased by cholecystectomy and CDD. We need to understand these issues to adequately advise patients of the implications of cholecystectomy and CDD. 


\section{Conclusion}

${ }^{99 m}$ Tc-HIDA scanning of the hepatobiliary system is a reasonable and reliable method for the quantitative evaluation of DGR. CDD is frequently associated with mild to moderate DGR compared to cholecystectomy alone group. Sever DGR is rarely seen. Most of the patients are asymptomatic and the degree of DGR does not necessarily produce symptoms in all patients. We need to understand these issues to adequately advise patients of the implications of cholecystectomy and CDD.

\section{References}

[1] Ritchie, W.P. (1994) Alkaline Reflux Gastritis. Gastroenterology Clinics of North America, 23, 281-294.

[2] Kuran, S., Parlak, E. and Aydog, G. (2008) Bile Reflux Index after Therapeutic Biliary Procedures. BMC Gastroenterology, 8, 4. http://dx.doi.org/10.1186/1471-230X-8-4

[3] Schweitzer, E.J., Harmon, J.W. and Bass, B.L. (1984) Bile Acid Reflux Precedes Mucosal Barrier Disruption in the Rabbit Esophagus. American Journal of Physiology, 247, G480-G485.

[4] Muhammed, I., Mclooughin, G.P. and Holt, S. (1980) Non-Invasive Estimation of Duodenogastric Reflux Using Technetium-99m p-Butyl-Iminodiacetic Acid. Lancet, 2, 1162-1165. http://dx.doi.org/10.1016/S0140-6736(80)92596-9

[5] Xyos, E., Vassilakis, J.S. and Fountos, A. (1991) Enterogastric Reflux after Various Type of Anti-Ulcer Gastreic Surgery: Quantitation by ${ }^{99 \mathrm{~m}}$ Tc-HIDA Scintigraphy. Gastroenterology, 101, 991-998.

[6] Stein, H.J., Smyrk, T.C., DeMeester, T.R., Rouse, J. and Hinder, R.A. (1992) Clinical Value of Endoscopy and Histology in the Diagnosis of Duodenogastric Reflux Disease. Surgery, 112, 796-803.

[7] Vaezi, M.F., Lacamera, L.R. and Richter, J.E. (1994) Validation Studies of Bilitec 2000: An Ambulatory Duodenogastric Reflux Monitoring System. American Journal of Physiology, 267, 1050-1057.

[8] Just, R.J., Leite, L.P. and Castell, D.O. (1996) Changes in Overnight Fasting Intragastric pH Show Poor Correlation with Duodenogastric Bile Reflux in Normal Subjects. American Journal of Gastroenterology, 91, 1567-1570.

[9] McDonnell, C.O., Bailey, I., Stumpf, T. and Walsh, T.N. (2002) The Effect of Cholecystectomy on Plasma Cholecystokinin. American Journal of Gastroenterology, 97, 2189-2192. http://dx.doi.org/10.1111/j.1572-0241.2002.05971.x

[10] Nassr, A.O., Gilani, S.N., Atie, M. and Abdelhafiz, T. (2011) Does Impaired Gallbladder Function Contribute to the Development of Barrett's Esophagus and Esophageal Adenocarcinoma? Journal of Gastrointestinal Surgery, 15, 908914. http://dx.doi.org/10.1007/s11605-011-1520-Z

[11] Nogi, K., Haruma, K., Taniguchi, H., Yomota, E., Okajima, M., Hananoki, M., et al. (2001) Duodenogastric Reflux Following Cholecystectomy in the Dog: Role of Antroduodenal Motor Function. Alimentary Pharmacology \& Therapeutics, 15, 1233-1238. http://dx.doi.org/10.1046/j.1365-2036.2001.01035.X

[12] Mearin, F.R.X., Balboa, A., Antolin, M., Varas, M.J. and Malagelada, J.R. (1995) Duodenogastric Bile Reflux and Gastrointestinal Motility in Pathogenesis of Functional Dyspepsia. Role of Cholecystectomy. Digestive Diseases and Sciences, 40, 1703-1709. http://dx.doi.org/10.1007/BF02212691

[13] Souza, R.F., Krishnan, K. and Spechler, S.J. (2008) Acid, Bile, and CDX: The ABCs of Making Barrett's Metaplasia. American Journal of Physiology: Gastrointestinal and Liver Physiology, 295, G211-G218. http://dx.doi.org/10.1152/ajpgi.90250.2008

[14] Gutschow, C.A., Bludau, M., Vallböhmer, D., Schröder, W., Bollschweiler, E. and Hölscher, A.H. (2008) NERD, GERD and Barrett's Esophagus: Role of Acid and Non-Acid Reflux Revisited with Combined pH-Impedance Monitoring. Digestive Diseases and Sciences, 53, 3076-3081. http://dx.doi.org/10.1007/s10620-008-0270-6

[15] Manifold, D.K., Anggiansah, A. and Owen, W.J. (2000) Effect of Cholecystectomy on Gastroesophageal and Duodenogastric Reflux. American Journal of Gastroenterology, 95, 2746-2750.

[16] Freedman, J., Ye, W., Näslund, E. and Lagergren, J. (2001) Association between Cholecystectomy and Adenocarcinoma of the Esophagus. Gastroenterology, 121, 548-553. http://dx.doi.org/10.1053/gast.2001.27217

[17] Hashimoto, N. (2014) Effects of Bile Acids on Cyclooxygenase-2 Expression in a Rat Model of Duodenoesophageal Anastomosis. World Journal of Gastroenterology, 20, 6541-6546. http://dx.doi.org/10.3748/wjg.v20.i21.6541 